\title{
Processos dependentes de informação em empresas incubadas e graduadas de base tecnológica: um estudo comparativo de casos
}

Thais Elaine Vick

\author{
Doutoranda em Engenharia de Produção pela \\ Escola de Engenharia de São Carlos, Universidade \\ de São Paulo (EESC-USP). Bolsista de Doutorado \\ do CNPq.
}

Marcelo Seido Nagano

\begin{abstract}
Professor Doutor do Departamento de Engenharia de Produção da Escola de Engenharia de São Carlos, Universidade de São Paulo - USP. Pós-doutor em Matemática e Computação Aplicada pelo Instituto Nacional de Pesquisas Espaciais. Pesquisador e Bolsista de Produtividade em Pesquisa do CNPq.
\end{abstract}

A competitividade, atualmente, introduz novos comportamentos e leva empresas a uma situação de desconforto e, muitas vezes, a não adaptação às exigências ambientais. Percebe-se um crescente número de desafios associados com o controle de informação em organizações com atividades de engenharia, em particular os volumes crescentes de informação de natureza continuamente mutável. Pode-se afirmar que 0 desempenho inovador de uma organização é diretamente proporcional a sua capacidade de gerenciar informações. Desta forma, a importância da gestão da informação é reconhecida em detrimento da procura de meios mais competentes para atender demandas atuais. $O$ artigo teve como propósito analisar doze processos dependentes de informação em empresas de base tecnológica, através das principais fases do processo de gerenciamento da informação. Optou-se pelo emprego do método comparativo de casos e pela pesquisa qualitativa, realizada em nove empresas de base tecnológica, as quais se encontravam incubadas ou que passaram recentemente pela fase de incubação no Parque Tecnológico da cidade de São Carlos, do estado de São Paulo. Entre os principais resultados, constatou-se que, nas empresas graduadas, a gestão da informação e seus procedimentos foram identificados de forma mais 
consciente e estruturados em relação às empresas incubadas.

Palavras-chave: Processo de Gerenciamento da Informação; Empresas de Base Tecnológica.

\section{Information-dependent processes in incubated and graduated technology- based companies: a comparative case study}

Nowadays, competitiveness introduces new behaviors and leads companies to a discomforting situation and often to non adaptation to environmental requirements. A growing number of challenges associated with control of information in organizations with engineering activities can be seen, particularly, the growing amount of information subject to continuous changes. The innovative performance of an organization is directly proportional to its ability to manage information. Thus, the importance of information management is recognized by the search for more competent ways to face current demands. The purpose of this article was to analyze informationdependent processes in technology-based companies, through the four major stages of information management. The comparative method of cases and qualitative research were used. The research was conducted in nine technology-based companies which were incubated or recently went through the incubating process at the Technological Park of São Carlos, in the state of São Paulo. Among the main results, it was found that in graduated companies information management and its procedures were identified as more conscious and structured in contrast to those of the incubated companies.

Keywords: Information Management Process; Technology-based Companies.

Recebido em 01.03.201 Aceito em 02.07.2011 


\section{Introdução}

Recentes estudos têm direcionado seus enfoques nos fenômenos informacionais a partir da perspectiva organizacional e dos sujeitos que produzem, organizam, recebem, disseminam e interpretam informações. Outros estudos têm envolvido questões sobre competência e comportamento informacional, seus modos de organização; processos de apropriação da informação; relações de trabalho; e sobre a relação tecnologia e trabalho. O escopo fundamental é o entendimento das organizações a partir dos fenômenos analisados e da natureza de interação dos processos que envolvem a informação.

O ponto de partida para estudos sobre processos dependentes de informação é a área de gestão da informação, que se inicia com a demanda de informação. O processo de atendimento a essa demanda envolve o estudo da informação e suas características, fluxos e necessidades. Já o processo de agregação de valor às informações, inclui habilidades, como análise, condensação, interpretação, representação e estratégias de busca e apresentação da informação disponibilizada, de acordo com os seus canais e suportes e, também, com os tipos de informações (MARCHIORI, 2002).

No que concerne aos processos informacionais, pode-se analisá-los de duas maneiras: primeiro, por discutir qual é o processo principal, identificando as atividades básicas que geram as demandas de informação. A segunda maneira é analisar processos mais específicos, particularmente dependentes da informação, ou seja, analisar o processo de gerenciamento da informação, composto de quatro passos genéricos: determinação das exigências, obtenção, distribuição e utilização da informação (DAVENPORT, 2001).

Uma metodologia eficiente deve ser estabelecida a fim de avaliar a informação, para evitar a sobrecarga e reter a informação correta e, assim, seja possível reutilizá-la. Atualmente, muitas ferramentas existem para avaliar recursos na engenharia, mas não são inteiramente associadas com a avaliação da informação. Enquanto a quantidade de informação disponível aumenta, sua qualidade transforma-se em um fator importante para a eficácia nas organizações e para os indivíduos. O desempenho de um indivíduo ou de uma organização pode ser prejudicialmente afetado pelo desequilíbrio de informação (EPPLER; MENGIS, 2004).

Muitas organizações estão cientes que estes problemas geram algumas perguntas fundamentais, como: quanta informação uma organização precisa? Que fragmentos de informação uma organização precisa? E quando a organização precisa desses? (EPPLER; MENGIS, 2004). Contudo, pode ser uma falha aprender de uma experiência antecedente, porque a informação não foi capturada ou não é prontamente recuperável em um contexto significativo. Encontrar respostas a estas perguntas é importante para todo o funcionamento da organização, em qualquer setor empresarial. 
Entretanto, dentro de setores altamente tecnológicos, inovadores e competitivos, a definição destas questões é particularmente crítica. Isso se deve pela natureza intensiva da informação de processos da engenharia. Responder a essas perguntas é imprescindível, pelo fato de haver uma combinação de processos criativos com as atividades em equipe e, também, dentro das organizações, que empreendem em grande parte das atividades (ZHAO et al., 2008).

Com base nas definições, o presente trabalho pretende promover uma discussão acerca do seguinte questionamento: em processos dependentes de informação, como se encontram as quatro principais fases do gerenciamento informacional em empresas de base tecnológica? Para tanto, são analisados doze processos dependentes de informação encontrados a partir das determinações de exigências, da obtenção, da distribuição e da utilização da informação pelas empresas.

\section{0 gerenciamento da informação: processo e desafios}

É a partir da década de 80, que a gestão da informação (GI) encontra maiores evidências de crescente importância na vida das organizações. Inicia-se uma trajetória que coloca a GI no mesmo nível dos demais trabalhos, como a gestão de recursos humanos, gestão de negócios, gestão de processos. Destarte, a GI passa a ser considerada mais uma atividade essencial, como qualquer outro tipo de trabalho de cunho organizacional.

O processo de gerenciamento da informação busca explicar o comportamento da organização, examinando os fluxos de informação em torno dela. Estudos precedentes, como o de Chou et al. (2007), mostraram que compreender a capacidade do processamento da informação é importante, porque facilita as atividades de criação de conhecimento e afetam, assim, as ações estratégicas. Da mesma forma, Gold et al. (2001) recomendam que a chave para compreender o sucesso e a falha da gestão do conhecimento consiste em identificar e em avaliar as condições prévias, tais como as capacidades de processo de informação, que são necessárias para que o esforço floresça.

A complexidade, que é atualmente característica das organizações, faz com que a exigência de habilidades ligadas ao gerenciamento da informação seja de total necessidade para um bom desempenho estratégico. A falta de controle da informação impede a sinergia entre os diferentes membros da organização (CÂNDIDO et al., 2005).

Davenport (2001) faz menção aos grupos que se preocupam com este processo, denominando-os inovadores de informação, ou seja, uma equipe com talento e criatividade que busca novas informações e maneiras inovadoras de apresentá-las. Esses fornecedores de informação podem criar novos bancos de dados para dar apoio aos negócios e fornecer informações interessantes sobre o mercado, que podem dar indicações sobre as condições de concorrentes. Em outras palavras, o lema é resolver problemas através do uso eficiente da informação ou abrir 
novas oportunidades. Uma abordagem participativa que enfatize objetivos estratégicos, juntamente com fatores externos como espaço físico comum e trabalho em equipe, é o que oferece os melhores resultados.

Consequentemente, faz-se necessário descobrir informações que indiquem qual o norte a ser seguido, qual o melhor caminho que propicie maior segurança para suas ações estratégicas.

A ideia de recurso estratégico nas organizações fez com que a informação passasse a ser gerenciada, tal como são gerenciados os recursos financeiros e humanos. Inicialmente denominada GRI (gestão de recursos informacionais), a gestão da informação representa toda a cadeia de valores da informação e, em outros termos, deve incluir a definição das necessidades de informação, a coleta, armazenamento, distribuição e recuperação, para a subseqüente utilização das informações (CIANCONI, 2003).

A Figura 1 apresenta o processo de gerenciamento da informação segundo Davenport (2001), que é utilizado como fundamento deste trabalho.

Figura 1 - O processo de gerenciamento da informação

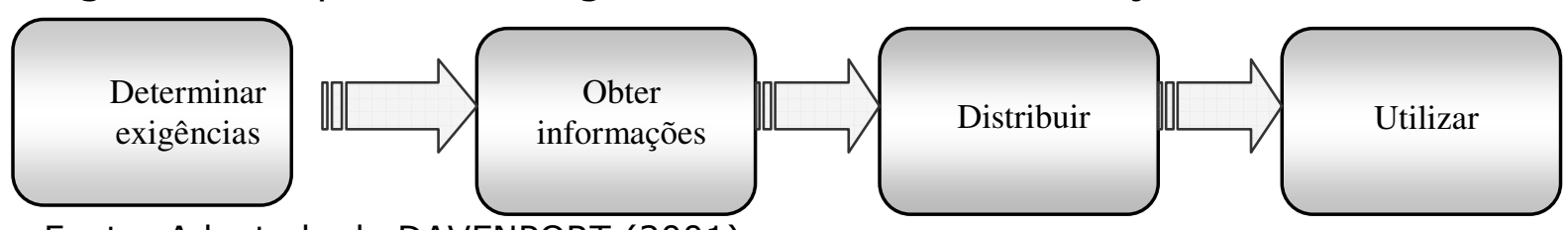

Fonte: Adaptado de DAVENPORT (2001).

São consideradas fases do processo:

a) determinação de exigências: combinar e conhecer as necessidades e exigências de informação das pessoas que as necessitam. É possível que os gestores de informação não saibam se uma determinada informação está disponível no ambiente interno ou externo da empresa, tampouco se ela pode ser gerada internamente. Nesse caso, os profissionais que lidam com a informação precisam, além de identificar as necessidades informativas da empresa, analisar se tais fontes estão disponíveis ou podem ser geradas, bem como divulgar as formas de acesso à informação;

b) obtenção: obter a informação, analisando seu conteúdo, de forma a estabelecer os níveis de qualidade, segurança e confiabilidade. Os usuários obtêm informações de muitas e diferentes fontes, formais e informais. As fontes informais (colegas de trabalho, contatos pessoais, etc.) são quase sempre tão ou mais importantes que as fontes formais (banco de dados online, intranet etc.);

c) distribuição: disseminar a informação ao grupo que a necessita. Obviamente, a interação humana, nessa fase, pode aumentar o valor estratégico da informação. O desafio reside em identificar e antecipar outras informações não previstas. Para isso, é necessário localizar um dispositivo de interface que estabeleça a ligação entre os usuários e os responsáveis pela distribuição e disseminação da informação; e 
d) utilização: de caráter bastante pessoal, é a maneira com que o profissional absorve e digere informações estruturadas e nãoestruturadas. Entende-se por informação estruturada aquela encontrada em documentos de ordem formal, como dados de desempenho operacional ou outras informações quantitativas. Informações nãoestruturadas englobam de notícias e ideias a rumores e conversas informais (DAVENPORT, 2001).

Em relação ao uso da informação, Choo (2003) acredita que é uma conceituação de difícil definição, porque envolve a seleção e o processamento das informações, de modo a solucionar um problema, responder perguntas, tomar decisões, negociar posições e entender situações. Para o autor, o resultado da utilização da informação é uma mudança no estado de conhecimento do indivíduo e de sua capacidade de ação.

Prusak e McGee (1994) afirmam que o gerenciamento da informação é um processo porque representa um conjunto de tarefas conectadas logicamente e deve ser genérico por duas razões:

1) porque a informação recebe ênfases diferentes em cada segmento econômico e em cada organização; e

2) porque as diferentes tarefas dentro do modelo assumem diferentes níveis de importância e valor entre as organizações.

Choo (1998) considera a importância dos indivíduos processadores de informação e enfatiza que as organizações a utilizam por formas distintas. Na primeira delas, a informação é coletada do ambiente e interpretada para a construção de significados. Na segunda forma, a organização compartilha informações almejando a inovação. Por último, utilizam-se essas informações procuradas, analisando-as para a tomada de decisões.

Choo (1998) constrói, ainda, um ciclo, como forma de representar a relação entre informação e conhecimento, no qual atrela a necessidade, a busca e o uso de informação, concomitantemente. Essas etapas compõem a estrutura cognitiva interna dos indivíduos e sua organização emocional:

necessidade de informação: representa elementos cognitivos, afetivos e situacionais, sentida também como uma incerteza. A necessidade de informação progressivamente vai chegando à consciência e então a questão é formalizada;

busca pela informação: a escolha das fontes de informação depende da inserção do indivíduo e da motivação que gerou a busca, através das etapas: iniciação, encadeamento, pesquisa, diferenciação, monitoramento, extração, verificação e conclusão. As três primeiras categorias são importantes para o desenvolvimento do foco e estratégia da pesquisa, as demais são fortemente influenciadas pelo ambiente cultural e organizacional; e

uso da informação: seleção e processamento de informação que resultará em um novo conhecimento. A informação, nessa etapa, é utilizada a fim de responder questões, resolver problemas, tomar 
decisões, negociar posições ou construir significados para determinada situação.

A GI, portanto, está condicionada às seguintes premissas: na criação de significados para o entendimento do seu ambiente de atuação; na construção de conhecimentos para suprir lacunas de conhecimento e desenvolver novas competências; e na tomada de decisões para escolher formas de ação. A geração e transformação da informação são moldadas pela cultura e pela especificação de regras, rotinas e papéis. Informações e significados são forjados nos pensamentos, sentimentos e ações dos indivíduos, em um processo cíclico (SILVEIRA; ODDONE, 2007).

Ao buscar e usar a informação, as pessoas estão continuamente construindo sentido a respeito do ambiente e, fazendo isso, parecem empregar estratégias básicas, dependendo do tipo de situação e das necessidades informacionais em que se encontram (CHOO, 1998; POPADIUK; CHOO, 2006).

O processamento da informação gera mais informação, que, por sua vez, gera conhecimento; que subsidia a estratégia fornecendo base para a ação; e que fornece a realimentação necessária para recomeçar o processo. Assim, a GI deve ser vista como a gestão de uma rede de processos que adquirem, criam, organizam, distribuem e usam a informação em um ciclo contínuo (CHOO, 1998; POPADIUK; CHOO, 2006).

\section{Metodologia}

De natureza aplicada, a pesquisa visa a gerar conhecimentos para aplicação prática na resolução de problemas específicos. De acordo com seus objetivos, é exploratória, com o propósito de aumentar o conhecimento sobre o problema com o intuito de torná-lo claro. De enfoque qualitativo, utiliza a coleta e análise de dados para responder às questões de pesquisa. De acordo com seus procedimentos técnicos, é caracterizada com um estudo comparativo de casos, já que se busca investigar em profundidade mais de uma empresa, propondo comparações entre elas.

Foram selecionadas nove empresas para o desenvolvimento da pesquisa. A escolha de empresas de base tecnológica deve-se à magnitude de informação e conhecimento nelas incorporados. A distribuição das empresas estritamente de base tecnológica configura-se da seguinte forma:

a) cinco empresas instaladas, pertencentes a duas incubadoras: Cinet e Softnet, do Parque Tecnológico da cidade de São Carlos, estado de São Paulo. As empresas incubadas foram nomeadas: A, B, C, D e E;

b) quatro empresas recentemente graduadas, que pertenceram às incubadoras Cinet e Softnet, do Parque Tecnológico da cidade de São Carlos, estado de São Paulo. As empresas graduadas foram nomeadas: $F$, G, H e I.

Entende-se por graduadas as organizações que passam pela incubação e alcançam desenvolvimento suficiente para ser habilitada a 
deixar a incubadora. No entanto, a empresa graduada pode manter o vínculo com o parque tecnológico na condição de empresa associada (ANPROTEC, 2004).

Utilizou-se um roteiro de entrevista com questionamentos estruturados, a fim de possibilitar uma comparação entre as respostas obtidas pelos pesquisados. Foram realizadas entrevistas individualmente, com os responsáveis pela GI das empresas selecionadas.

Através das diretrizes de Bardin (1979), a análise dos dados desta pesquisa constituiu-se da seguinte forma:

1) pré-análise: através da leitura flutuante do diário de campo e das transcrições das entrevistas, formularam-se os indicadores que fundamentaram a interpretação final.

2) exploração do material: definiram-se:

a) categoria: o grande tema de pesquisa - o processo de gerenciamento da informação;

b) unidades de registro: os subtemas: determinação de exigências, obtenção, distribuição, utilização; e

c) regras de contagem: foram estabelecidas pela presença dos elementos mais significativos.

3) tratamento dos resultados: na inferência, utilizou-se das entrevistas transcritas e na interpretação dos dados houve a exposição de conceitos teóricos e reflexões abrangentes sobre o setor em questão.

\section{Caracterização do setor pesquisado}

Torkomian et al. (2006) apresentaram uma avaliação das áreas de potencial científico e tecnológico em São Carlos, a partir dos cursos de graduação e programas de pós-graduação ofertados, das empresas de base tecnológica instaladas e dos projetos aprovados em programas de financiamento de P\&D da FAPESP. Na avaliação das empresas de base tecnológica (EBTs), foi realizado um levantamento para identificar a quantidade e a área tecnológica em que se concentra a operação de cada empresa. O resultado foi uma listagem com 112 EBTs em São Carlos, que se concentram nas áreas de computação, eletrônica, engenharia biomédica, materiais avançados, mecânica, serviços de engenharia, ótica e química, conforme Tabela 1. 
Tabela 1 - Campos tecnológicos das atividades das EBTs de São Carlos

\begin{tabular}{c|c}
\hline Campos Tecnológicos & EBTs \\
\hline Computação & $38 \%$ \\
Eletrônica & $18 \%$ \\
Engenharia Biomédica & $10 \%$ \\
Materiais avançados & $09 \%$ \\
Mecânica & $08 \%$ \\
Serviços de Engenharia & $07 \%$ \\
Ótica & $06 \%$ \\
Química & $03 \%$ \\
Outros & $01 \%$ \\
\hline
\end{tabular}

Fonte: Adaptado de PIEKARSKI (2007).

As empresas selecionadas para esta pesquisa correspondem aos seguintes campos tecnológicos:

Tabela 2 - Campos tecnológicos das EBTs selecionadas

\begin{tabular}{c|c}
\hline Campos Tecnológicos & Empresa \\
\hline Eletrônica & A \\
Computação & B \\
Materiais avançados & $\mathrm{C}$ \\
Mecânica & $\mathrm{D}$ \\
Serviços de Engenharia & $\mathrm{E}$ \\
Eletrônica e serviços de engenharia & $\mathrm{F}$ \\
Computação & $\mathrm{G}$ \\
Engenharia biomédica & $\mathrm{H}$ \\
Ótica & $\mathrm{I}$ \\
\hline
\end{tabular}

Fonte: Dados da pesquisa.

\section{Comparações sobre os processos de GI}

Os processos de GI foram observados como fatores propulsores das empresas graduadas ( $F, G, H$ e I) e como fatores restritivos das empresas incubadas ( $A, B, C, D$ e $E)$. As tabelas, a seguir, trazem uma síntese dos grandes temas estudados e dos registros obtidos. Conforme tabela 3, verificam-se as comparações das nove empresas com relação à determinação de exigências de informação. Na tabela 3, foram identificados como processos dependentes de informação: 1) as áreas para as quais os funcionários mais precisam de informações; 2) o sistema de informações vigente e sua adequação para a agilidade dos serviços; 3 ) a disponibilidade e atualização das informações para procedimentos 
diários; e 4) a identificação de um setor ou pessoa específica para o processamento de informações externas:

Tabela 3 - Comparação da determinação das exigências de informação nas empresas

\begin{tabular}{|c|c|c|c|c|}
\hline \multicolumn{5}{|c|}{ DETERMINAÇÃO DAS EXIGÊNCIAS DE INFORMAÇÃO } \\
\hline \multirow{2}{*}{ Teoria } & \multicolumn{4}{|c|}{ Combinar e conhecer as necessidades e exigências de informação das pessoas que as necessitam } \\
\hline & $\begin{array}{l}\text { Inovação e } \\
\text { tecnologia }\end{array}$ & $\begin{array}{c}\text { Sistema de } \\
\text { informações } \\
\text { adequado }\end{array}$ & Disponibilidade/atualização & Setor ou pessoa específica \\
\hline Empresa A & $\mathrm{X}$ & & & \\
\hline Empresa B & $\mathrm{X}$ & & $\mathrm{X}$ & \\
\hline Empresa C & $\mathrm{X}$ & & $\mathrm{X}$ & \\
\hline Empresa D & $\mathrm{X}$ & $\mathrm{X}$ & $\mathrm{X}$ & \\
\hline Empresa E & $\mathrm{X}$ & & & \\
\hline Empresa F & $\mathrm{X}$ & $\mathrm{X}$ & $\mathrm{X}$ & $\mathrm{X}$ \\
\hline Empresa G & $\mathrm{X}$ & $\mathrm{X}$ & $X$ & \\
\hline Empresa H & $\mathrm{X}$ & $\mathrm{X}$ & $X$ & $\mathrm{X}$ \\
\hline $\begin{array}{l}\text { Empresa } \\
\text { I }\end{array}$ & $x$ & $x$ & $x$ & $x$ \\
\hline
\end{tabular}

Fonte: Dados da pesquisa.

As empresas $A, B$, e $E$ possuem sistemas de informações viáveis, porém não são considerados mais adequados para a agilidade dos serviços. O sistema da empresa $\mathrm{C}$ só diz respeito a informações financeiras.

A disponibilidade e atualização das informações acontecem na maioria das empresas. Na empresa $\mathrm{C}$, apenas as informações sobre capital, custo de produção, de vendas e despesas estão atualizadas.

Apenas as empresas $\mathrm{F}, \mathrm{H}$ e I possuem um setor ou pessoa específica para a coleta de informações sobre o ambiente externo.

A tabela 4 traz a comparação da obtenção da informação nas empresas. Nela, foram identificados como processos dependentes de informação: 5) a realização de reuniões frequentes para discutir o que a empresa pode precisar no futuro; 6) orientação por parte dos funcionários para detectar mudanças específicas no setor; 7) as pesquisas sobre fontes internas de informação; e8) as pesquisas sobre fontes externas de informação: 
Tabela 4 - Comparação da obtenção da informação nas empresas

\begin{tabular}{|c|c|c|c|c|}
\hline \multicolumn{5}{|c|}{ OBTENÇÃO DA INFORMAÇÃO } \\
\hline \multirow{2}{*}{ Teoria } & \multicolumn{4}{|c|}{$\begin{array}{l}\text { Obter a informação analisando seu conteúdo de forma a estabelecer os níveis de } \\
\text { qualidade, segurança e confiabilidade. Os usuários obtêm informações diferentes } \\
\text { fontes, formais e informais. }\end{array}$} \\
\hline & $\begin{array}{c}\text { Reuniões sobre } \\
\text { futuro }\end{array}$ & $\begin{array}{c}\text { Orientação para } \\
\text { detectar mudanças }\end{array}$ & Fontes internas & Pesquisas / fontes externas \\
\hline Empresa A & $X$ & & $\mathrm{X}$ & \\
\hline Empresa B & $\mathrm{X}$ & & $\mathrm{X}$ & \\
\hline Empresa C & & $\mathrm{X}$ & $\mathrm{X}$ & $\mathrm{X}$ \\
\hline Empresa D & $\mathrm{X}$ & $\mathrm{X}$ & $X$ & \\
\hline Empresa E & $\mathrm{X}$ & & $\mathrm{X}$ & $\mathrm{X}$ \\
\hline Empresa F & & & $\mathrm{X}$ & $\mathrm{X}$ \\
\hline Empresa G & $\mathrm{X}$ & $\mathrm{X}$ & $\mathrm{X}$ & $\mathrm{X}$ \\
\hline Empresa $\mathrm{H}$ & $\mathrm{X}$ & $\mathrm{X}$ & $\mathrm{X}$ & $\mathrm{X}$ \\
\hline Empresa I & $\mathrm{X}$ & $\mathrm{X}$ & $\mathrm{X}$ & $\mathrm{X}$ \\
\hline
\end{tabular}

Fonte: Dados da pesquisa.

As pesquisas feitas nas empresas $C$ e $E$ sobre produtos que a empresa pode precisar são feitas informalmente. A empresa $F$ faz pesquisas sobre projeção de produtos e a empresa $G$ possui pesquisas somente na área de produtos, o que não acontece na área de serviços.

A empresa $F$ encontra dificuldades em orientar seus funcionários para detectar mudanças fundamentais e preferências do mercado por produtos. A empresa I orienta o departamento de P\&D e vendedores. Apenas nas empresas C e $F$ não são feitas reuniões a fim de descobrir quais produtos ou serviços podem-se precisar no futuro.

A tabela 5 traz a comparação da obtenção da informação nas empresas. Nela, foram identificados como processos dependentes de informação: 9) encontros interdepartamentais; 10) distribuição imediata de informações sobre o desenvolvimento de um novo produto; 11) produção de documentos de ordem quantitativa e formal; e 12) rotinas documentadas e padronizadas: 
Tabela 5 - Comparação da distribuição e da utilização da informação nas empresas

\begin{tabular}{|c|c|c|c|c|}
\hline \multicolumn{5}{|c|}{ DISTRIBUIÇÃO E UTILIZAÇÃO DA INFORMAÇÃO } \\
\hline \multirow{2}{*}{ Teoria } & \multicolumn{4}{|c|}{$\begin{array}{l}\text { Disseminar a informação ao grupo que a necessita. A interação humana nessa fase pode } \\
\text { aumentar o valor estratégico da informação. A utilização tem caráter bastante pessoal, é a } \\
\text { maneira com que o profissional absorve e digere informações. }\end{array}$} \\
\hline & $\begin{array}{c}\text { Encontros } \\
\text { interdepartamentais }\end{array}$ & $\begin{array}{l}\text { Info. imediata } \\
\text { sobre } \\
\text { desenvolvimento } \\
\text { de produtos }\end{array}$ & $\begin{array}{l}\text { Documentos de } \\
\text { ordem formal }\end{array}$ & $\begin{array}{l}\text { Rotinas documentadas e } \\
\text { padronizadas }\end{array}$ \\
\hline Empresa A & & $\mathrm{X}$ & & \\
\hline Empresa B & $\mathrm{X}$ & & $\mathrm{X}$ & $\mathrm{X}$ \\
\hline Empresa C & $\mathrm{X}$ & $\mathrm{X}$ & & \\
\hline Empresa D & $\mathrm{X}$ & $\mathrm{X}$ & & \\
\hline Empresa E & & $\mathrm{X}$ & $\mathrm{X}$ & \\
\hline Empresa F & $\mathrm{X}$ & $\mathrm{X}$ & $\mathrm{X}$ & \\
\hline Empresa G & & $\mathrm{X}$ & $\mathrm{X}$ & $\mathrm{X}$ \\
\hline Empresa $\mathrm{H}$ & $\mathrm{X}$ & $\mathrm{X}$ & $\mathrm{X}$ & $\mathrm{X}$ \\
\hline Empresa I & $\mathrm{X}$ & $X$ & $X$ & $X$ \\
\hline
\end{tabular}

Fonte: Dados da pesquisa.

Nas empresas $\mathrm{C}, \mathrm{D}, \mathrm{F}$ e $\mathrm{H}$, os funcionários da engenharia de produto são os primeiros a saber quando algo importante acontece no processo de desenvolvimento de produtos e (ou) serviços. A empresa $G$ tem documentadas e padronizadas as rotinas apenas da área administrativa. $\mathrm{Na}$ empresa $\mathrm{E}$, são produzidos documentos de ordem formal apenas por exigências da consultoria que recebe. Os encontros para discutir tendências na indústria de serviços de informação são considerados interdepartamentais nas empresas D, F, H e I. Nas empresas B e C, esses encontros também são realizados, porém entre os funcionários, de forma não departamentalizada, por se tratar de empresas pequenas. A empresa E só realiza esse tipo de encontro com a consultoria.

\section{Propostas de melhores práticas para as empresas}

Baseando-se na análise dos dados coletados nas entrevistas, nos fatores propulsores e restritivos de cada empresa e nas tabelas que contêm a síntese da análise comparativa dos casos, percebeu-se uma significativa distinção entre as empresas recentemente graduadas e as empresas que ainda encontram-se na fase de incubação. Nas empresas incubadas $A, B, C, D$ e $E$, encontrou-se um maior nível de informalidade com relação ao gerenciamento da informação. Por esse motivo, elaborouse um quadro que contêm as principais sugestões de melhoria para as empresas, para as quais uma maior atenção deve ser dada. O quadro 1 
traz as propostas de melhores práticas para as empresas $A, B, C, D$ e $E$, com relação à determinação de exigências, obtenção, distribuição e utilização da informação:

Quadro 1 - Propostas de melhores práticas para as empresas A, B, C, D e $\mathrm{E}$

\begin{tabular}{|c|c|c|c|c|}
\hline $\begin{array}{c}\text { Empresas } \\
\text { A, B, C, D, E }\end{array}$ & $\begin{array}{c}\text { Determinação de } \\
\text { exigências }\end{array}$ & Obtenção & Distribuição & Utilização \\
\hline $\begin{array}{l}\text { Propostas de } \\
\text { melhores práticas }\end{array}$ & $\begin{array}{l}\text { Designação de } \\
\text { funcionário } \\
\text { específico } \\
\text { responsável pela } \\
\text { coleta de } \\
\text { informações } \\
\text { e pesquisas sobre } \\
\text { produtos e (ou) } \\
\text { serviços que a } \\
\text { empresa pode } \\
\text { precisar }\end{array}$ & $\begin{array}{l}\text { Produção e } \\
\text { controle de } \\
\text { documentos de } \\
\text { ordem formal; } \\
\text { preocupação com a } \\
\text { informalidade, a } \\
\text { disponibilidade e } \\
\text { atualização de } \\
\text { documentos } \\
\text { advindos de fontes } \\
\text { internas e externas }\end{array}$ & $\begin{array}{l}\text { Implantação de um } \\
\text { sistema } \\
\text { informatizado de } \\
\text { informações que } \\
\text { seja considerado o } \\
\text { mais adequado para } \\
\text { a agilidade dos } \\
\text { serviços }\end{array}$ & $\begin{array}{l}\text { Agrupamento e } \\
\text { sistematização de } \\
\text { documentos com } \\
\text { resultados e } \\
\text { aprendizados, } \\
\text { ligando-se a outros } \\
\text { documentos de } \\
\text { interesses } \\
\text { relacionados }\end{array}$ \\
\hline
\end{tabular}

Fonte: Dados da pesquisas

\section{Considerações finais}

Nos processos dependentes de informação das empresas incubadas, percebeu-se que existe maior preocupação com o registro das informações quantitativas e as informações qualitativas e informais, embora detectadas pelas empresas, não possuem um tratamento diferenciado. As empresas utilizam as informações disponíveis e nem sempre contam com todas as informações necessárias. Valorizam-se as informações pessoais. Quando encontrados, os sistemas de informações não são satisfatórios para um efetivo gerenciamento estratégico da informação.

Diferentemente, nas empresas graduadas, faz-se uso de maiores fontes formais de informação e há maior preocupação com a sua organização e padronização. Os sistemas de informações encontrados foram considerados essenciais e satisfatórios para as necessidades informacionais internas.

As diferenças nos resultados encontrados entre empresas incubadas e recentemente graduadas são, em grande parte, devido ao ambiente de incubação, que prioriza os estímulos à criação de conhecimento, no intuito de proporcionar maiores condições de aprendizagem para 0 desenvolvimento das empresas incubadas, e estas, por sua vez, têm a oportunidade de aprender em condições privilegiadas. Neste ambiente, a geração de conhecimentos passíveis de serem transformados em novos produtos e processos está entre seus objetivos principais, ao passo que a GI aparece em seus objetivos secundários.

O processo inovador existente tanto nas empresas incubadas quanto nas empresas graduadas deve ser direcionado por dois eixos conceituais: 
o envolvimento dos indivíduos quanto à informação e o envolvimento quanto ao conhecimento. As capacidades de GI, como vistas no início deste trabalho, devem ser identificadas e avaliadas como condições prévias à criação de conhecimento.

Ademais, sugerem-se pesquisas futuras, de natureza aplicada, que auxiliem organizações a lidar com o tratamento da informação, tornandoas, cada vez mais, capazes de apropriar-se do conhecimento buscado e transformá-lo em produtos e serviços.

\section{Referências}

ASSOCIAÇÃO NACIONAL DE ENTIDADES PROMOTORAS DE EMPREENDIMENTOS INOVADORES (ANPROTEC). Panorama 2004. Brasília: ANPROTEC, 2004.

BARDIN, L. Análise de conteúdo. Portugal: Edições 70, 1979.

CÂNDIDO, C. A. et al. Gestão estratégica da informação: semiótica aplicada ao processo de tomada de decisão. Datagramazero, Rio de Janeiro, v. 6, n. 3, p. 1-16, 2005.

$\mathrm{CHOO}, \mathrm{C}$. W. The knowing organization: how organizations use information to construct meaning, create knowledge, and make decisions. New York: Oxford Univerty, 1998.

$\mathrm{CHOO}, \mathrm{C}$. W. Information management for the intelligent organization: the art of scanning the environment. 3 ed. Medford, New Jersey: Information Today, 2003.

CHOU, T. C. et al. A path model linking organizational knowledge attributes, information processing capabilities, and perceived usability. Information \& Management, v. 44, p. 408-417, 2007.

CIANCONI, R. B. Gestão do conhecimento: visão de indivíduos e organizações no Brasil. 2003. 298 f. Tese (Doutorado em Ciência da Informação) - Instituto Brasileiro de Informação em Ciência e Tecnologia (IBICT), Rio de Janeiro, 2003.

DAVENPORT, T. H. Ecologia da informação: por que só a tecnologia não basta para o sucesso na era da informação. São Paulo: Futura, 2001.

EPPLER, M. J.; MENGIS, J. The concept of information overload: a review of literature from organization science, accounting, marketing, MIS, and related disciplines. The Information Society, v. 20, n. 5, p. 325-344, 2004.

GOLD, A. H. et al. Knowledge management: an organization capabilities perspective. Journal of Management Information Systems, v. 18, n. 1, p. $185-214,2001$.

MARCHIORI, P. A ciência da informação: compatibilidades no espaço profissional. Caderno de Pesquisas em Administração, São Paulo, v. 9, n. 1, p. 91-101, 2002. 
PIEKARSKI, A. E. T. O Sistema de Inovação em São Carlos sob uma abordagem sistêmica e a análise de redes. 2007. 244p. Tese (Doutorado em Engenharia de Produção) - Programa de Pós Graduação em Engenharia de Produção, Universidade Federal de São Carlos, 2007.

POPADIUK, S.; CHOO, C. W. Innovation and knowledge creation: how are these concepts related? International Journal of Information Management, v. 26, p. 301-311, 2006.

PRUSAK, L.; MCGEE, J. Gerenciamento estratégico da informação. Rio de Janeiro: Campus, 1994.

SILVEIRA, M. M.; ODDONE, N. Information needs and information behavior: concepts and models. Ciência da Informação: Brasília, v. 36, n. 1, p. 118-127, maio/ago. 2007.

TORKOMIAN, A. L. V. et al. A dinâmica de geração e uso do conhecimento em São Carlos: um estudo através das áreas de potencial científico e tecnológico. In: SEMINÁRIO NACIONAL DA ANPROTEC, 16, 2006, Salvador. Anais... Brasília: Associação Nacional de Entidades Promotoras de Empreendimentos Inovadores, 2006.

$\mathrm{ZHAO}, \mathrm{Y}$. et al. High value information in engineering organizations. International Journal of Information Management, v. 28, p. 246-258, 2008. 